\title{
O idoso doador de sangue e o cuidado da enfermagem hemoterápica ${ }^{1}$
}

The elderly blood donor and the hemotherapic nurse's care

\author{
El anciano donante de sangre y el cuidado de la enfermería de la hemoterapia
}

Nereida Lúcia Palko dos Santos', Marluci Andrade Conceição Stipp"', J osète Luzia Leite" "', Alessandra Sant' Anna Nunes ${ }^{\mathrm{IV}}$

\section{RESUMO}

O estudo destaca o cuidado da enfermagem hemoterápica aos idosos candidatos à doação de sangue, que é um ato responsável e representativo de autonomia, maturidade, e participação social a ser cultivado por toda a vida. Especial atenção e incentivo deve ser oferecido ao idoso, hígido, cidadão, que pode doar sangue, em uma população que galga a longevidade de forma saudável. O objetivo da reflexão proposta neste estudo é discutir sobre - cuidado da enfermagem hemoterápica ao idoso doador de sangue. As enfermeiras que realizam a Triagem Clínica em Hemoterapia estão envolvidas no cuidado de enfermagem aos doadores de sangue, em uma área de saúde especializada, e este cuidado envolve aspectos culturais, sociais, econômicos, de cidadania, da fisiopatologia, da qualidade de vida e do processo saúde-doença, devendo atender às diretrizes do Sistema Único de Saúde, do Ministério da Saúde, e estar articulado às Necessidades de Saúde dos candidatos a doadores de sangue idosos em seu desenvolvimento e envelhecimento.

Palavras chave: Enfermagem Geriátrica; Saúde do idoso; Serviço de Hemoterapia.

\footnotetext{
ABSTRACT

This study focuses on the hemotherapic nurse's care to the elderly blood donors. Blood donation is an act of autonomy, maturity and social responsibility, to be done during the individual's whole life. Brazilian population is living a healthy aging process, and requires attention, orientation and education to be blood donors in all ages. Our theoretical reflection is about elderly blood donors as hemotherapic nurse's clients. The objective of the study is a discussion about hemotherapic nurse's care to elderly blood donor. Nurses that works in the screening blood donor process are taken care of the population, as they are committed with the care of blood donors. The nursing care of elderly blood donor involving cultural, social-economic,
}

better quality of life and disease-health process, should consider the health needs of the elderly blood donors in a perspective of care, attending to the Unic Health Sistem and the Ministery of Health dispositions.

Key words: Geriatric Nursing; Helth of Elderly; Hemotherapy Service.

\section{RESUMEN}

Estudio reflexivo sobre el cuidado de la enfermería de la hemoterapia al anciano donante de sangre. El objetivo del estudio es discutir sobre el cuidado de enfermería del anciano donante de sangre. El cuidado de enfermería en el selecíon clínica y hematológica en el hemoterapia de la población deben envolver medidas educativas, de orientación, de encaminamineto y de prevención de agravamentos, siendo una posibilidad de acercamiento de la población con los servicios de salud. Proponemos una reflexión sobre la clarificación y la sensibilización referente al acto de donar sangre, responsable y símbolo de la autonomía, de la madurez, de la participación social con la especial atención y incentivo al anciano activo, poseedor de los derechos del ciudadano en suyas necesidades de la salud y de las determinaciones del Ministerio de la Salud y del Sistema Único de la Salud.

\footnotetext{
1 Trabalho originado a partir da elaboração do projeto de pesquisa da Tese de Doutorado: "As necessidades de saúde dos candidatos a doadores de sangue na triagem clínica em hemoterapia", desenvolvido na Escola de Enfermagem Anna Nery / UFRJ

Enfermeira, Doutoranda em Enfermagem da EEAN / UFRJ, Docente de Enfermagem da EEAN/UFRJ, Rio de Janeiro. Email: nereidasantos@oi.com.br

"Enfermeira, Doutora em Enfermagem, Docente de Enfermagem da EEAN/UFRJ, Rio de Janeiro. E-mail: marlustipp@gmail.com.

III Enfermeira, Doutora em Enfermagem, Docente Permanente de Enfermagem da EEAN/UFRJ, Pesquisadora 1A CNPq. Rio de Janeiro. E-mail: joluzia@gmail.com.

iv Enfermeira, Mestre em Enfermagem, Docente de Enfermagem da UERJ, Rio de Janeiro. Email:
} asantnunes@yahoo.com.br. 
Palabras clave: Enfermería Geriátrica; Salud del Anciano; Servicio de Hemoterapia.

\section{A DOAÇÃo de SANGUE NA TERCEIRA IDADE}

Nas últimas décadas ocorreu uma crescente expansão da população idosa no Brasil, em que o efeito combinado da redução dos níveis de fecundidade, e da mortalidade da população, resultará na transformação da pirâmide etária, com o formato tipicamente triangular, com base alargada, que cederá lugar a uma pirâmide populacional característica de franco envelhecimento na projeção da população do País por sexo e idade para o período $1980-2050^{(1)}$.

A projeção do envelhecimento da população ao longo dos próximos quarenta e dois anos poderá gradativamente se refletir no perfil dos candidatos a doadores de sangue, favorecendo um possível aumento no número de doadores idosos, entre sessenta e sessenta e cinco anos, uma vez que se projeta que a partir dos resultados analisados sobre o perfil populacional das próximas décadas, haverá significativas alterações na participação relativa de crianças e jovens na sociedade, acompanhadas do aumento proporcional dos adultos e, particularmente, dos idosos, que acessam diversas modalidades de serviços ${ }^{(1)}$.

Os Serviços de Hemoterapia precisam da participação da população saudável para doar altruística e voluntariamente o sangue e seus componentes, cabendo conhecer e estar integrada ao potencial perfil de doadores idosos que realizarão o ato cidadão da doação de sangue. A Norma Técnica Brasileira em Hemoterapia revisada em $2004^{(2)}$, possibilita que as pessoas idosas realizem doações de sangue, caracterizando um avanço para a hemoterapia e para a gerontologia, já que o envelhecimento populacional merece especial atenção dos formuladores de políticas públicas.

Com os avanços no conhecimento e entendimento sobre o envelhecimento, outros parâmetros além do cronológico são indicadores das condições de saúde da população idosa no Brasil $^{(3)}$. Ao tentar definir a velhice, devemos entender a sua transcendência biológica, física, mental e cultural. É complexo definir o começo da velhice, pois varia de acordo com as épocas e lugares. O indivíduo é condicionado pela atitude prática e ideológica da sociedade em relação a ele para identificar-se idoso(4). Ser e, estar idoso é também uma questão de "visão de mundo", que pode caracterizar um grande abismo entre a cronologia dos anos vividos e a relação do indivíduo com a sociedade e com sua família nos anos percorridos a partir de um marco etário determinado.

Além de serem produtores de atividades econômicas, intelectuais, afetivas e de bens socialmente mensuráveis e/ou perceptíveis para a vida, os idosos do Século XXI desenvolvem suas atividades de vida diária (AVD) e as atividades instrumentais da vida diária (AIVD) com desenvoltura, autonomia e independência por anos consecutivos. Quanto mais familiar e socialmente integrados estes estejam, e possuam higidez e condições estabelecidas para tanto ${ }^{(3)}$, é possível que continuem doando sangue por um período de até cinco anos de suas vidas, uma vez que a legislação(5) brasileira determina que a pessoa é idosa a partir dos sessenta anos, e que a Norma Técnica em Hemoterapia ${ }^{(2)}$ determina o limite etário máximo para a doação de sangue aos sessenta e cinco anos, onze meses e vinte e nove dias.

Há uma maior perspectiva acerca da higidez e integridade física do idoso, que em condições gerais pode chegar à velhice controlando e contornando o desgaste do corpo e da mente, principalmente, quando se investe na prevenção e controle das patologias ao longo da vida(3). Desta forma, passa a ser possível, desde 2002, a doação de sangue do idoso entre 60 e 65 anos de idade, a partir de uma criteriosa avaliação clínica e hematimétrica, com maior intervalo entre as doações, e uma freqüência máxima de até duas doações ao ano, permitindo que $o$ ato cidadão de doar sangue faça parte da vida adulta em todas as suas fases, desde que as integridades física e mental estejam preservadas, e patologias, como as crônicas, controladas ${ }^{(2)}$.

O cuidado de enfermagem a doadores de sangue idosos é uma prática recente, dos últimos seis anos, que se adapta às 
heterogêneas e complexas características do envelhecimento da população brasileira, e caracteriza um perfil específico de clientes, em uma área pouco explorada em estudos científicos. A pesquisa em bancos de dados (Biblioteca Virtual em Saúde: SciELO, MEDLINE, LILACS; BDEnf, entre outros) no período de 2002, ano que a doação de sangue de pessoas entre sessenta e sessenta e cinco anos é legalmente determinada ${ }^{(6)}$, até 2008 , com os unitermos: idoso, enfermagem, hemoterapia e doação de sangue, revelou apenas um trabalho em que é investigado pelas autoras o significado que doadores e receptores atribuem ao sangue ${ }^{(7)}$, demonstrando a escassez de trabalhos científicos sobre o tema.

Idosos são clientes da hemoterapia, como doadores, e não apenas como receptores de sangue e seus componentes, ou como pessoas supostamente doentes, são pessoas supostamente saudáveis doando "vida". A recente vertente que passou a existir para a relação da hemoterapia com a gerontologia, e os elos da equipe multidisciplinar, concretiza a necessidade de discutir a gerontologia e suas nuances neste espaço micropolítica de saúde ${ }^{(8-}$ 9).

Nos serviços de hemoterapia as atividades são desenvolvidas por profissionais da equipe multidisciplinar, que é composta por farmacêuticos, biólogos, médicos, enfermeiros e técnicos de enfermagem e de laboratório, entre outros. Os enfermeiros participam como membros da equipe multiprofissional, contribuindo efetivamente na elaboração e viabilização de uma assistência às pessoas que doam $e$ as que recebem sangue e seus componentes $^{(10)}$. Devem estar capacitados a desenvolver um olhar e uma prática gerontológica, com vistas a integrar o conjunto de ações que contribuem para a longevidade com qualidade de vida dos doadores de sangue idosos, além de garantir a segurança aos receptores dos componentes a serem transfundidos, em todo o ciclo do sangue.

Entender o cuidado de enfermagem ao idoso doador de sangue determina entender o ciclo do sangue em hemoterapia como um campo de ação de enfermagem que envolve: captação de doadores; triagem clínica; coleta do sangue; preparo; fracionamento do sangue e armazenamento dos seus componentes; testes imuno-hematológicos; preparo da transfusão; transfusão; hemovigilância; e controle do processo de distribuição (checagem e rechecagem sorológica, com o envio e recebimento nas unidades transfusionais).

Considerando os aspectos discutidos até o momento, coloca-se a seguinte situação problema deste trabalho: A enfermagem que atua em hemoterapia necessita de uma abordagem gerontológica no cuidado prestado ao doador de sangue idoso? A partir desta temos como objetivo discutir sobre o cuidado da enfermagem hemoterápica ao idoso doador de sangue.

\section{O CUI DADO DE ENFERMAGEM AO DOADOR DE SANGUE I DOSO}

Os profissionais de enfermagem que trabalham em hemoterapia, conhecendo o fenômeno biológico do envelhecimento, e cuidando dos idosos que doam sangue, ou candidatam-se a doar, devem considerar as Necessidades de Saúde da clientela, à luz da integralidade, da senescência, e das políticas de saúde vigentes como princípios inerentes ao cotidiano de cuidar.

Não podendo considerar apenas a idade cronológica como determinante da velhice, outras variáveis que compõem a vida da pessoa idosa devem ser consideradas, tais como: sexo, classe social, saúde, educação e outros que resultam na idade funcional, ou do grau de conservação do nível de capacidade adaptativa para as situações do dia-a-dia, o que fará com que pessoas idosas de uma mesma faixa etária tenham diferenças no processo de envelhecimento. Umas o terão com tranqüilidade, e outras cumprindo as alterações deste processo de forma mais tensa, abrupta ou até penosa ${ }^{(3-4)}$.

Discutir sobre o cuidado de enfermagem aos doadores de sangue idosos envolve saberes e fazeres de atuação em uma área de especialidade, onde, uma postura gerontológica de cuidado deve minimamente constar da geriatria preventiva, com ações para diminuir ou retardar o aparecimento de disfunções e morbidades crônico-degenerativas associadas à idade (prevenção primária); o estabelecimento das revisões anuais contribuindo na detecção 
precoce de doenças (prevenção secundária), e para os indivíduos já afetados pelas doenças, a adoção de programas de reeducação do estilo de vida e de prevenção que garantam estabilização da saúde (prevenção terciária) ${ }^{(3)}$.

Com mudanças progressivas na terceira idade, ocasionando, por exemplo, reduções na capacidade funcional, mudanças físicas e / ou fisiológicas, sociais, econômicas, afetivas, e pessoais no envelhecimento, para a enfermeira que atua na Triagem em Hemoterapia a compreensão das Necessidades de Saúde das pessoas, em um cuidado de enfermagem para a "doação da vida", pode favorecer e enriquecer o processo da doação de sangue e em particular da relação que se desenvolve no breve momento de encontro da enfermeira com o doador de sangue.

As Necessidades de Saúde são determinadas ou constituídas social e historicamente, só podendo ser captadas e trabalhadas em sua dimensão individual. No momento do encontro da enfermeira com o cliente na Triagem em Hemoterapia pode ser constituído este espaço, mas para tanto, seria quase inevitável a adoção de um conceito de Necessidades de Saúde que possa ter uma tradução e uma reconceitualização que se aproxime o mais fielmente possível da expressão dialética do individual e do social ${ }^{(11)}$.

As Necessidades de Saúde aqui discutidas, não são as Necessidades Humanas Básicas amplamente difundidas na enfermagem a partir de Wanda Horta, mas sim, as que perpassam por uma interação contínua das esferas individuais e coletivas dos sujeitos, sendo que é o indivíduo quem vivencia o processo de determinação de suas carências (necessidades), mediante escolhas, que são determinantes para opções entre valores e modos de vida, colocando em pauta a ampliação do entendimento da enfermagem hemoterápica ao considerar a expressão dos candidatos a doadores quanto as suas necessidades e o cuidado que demandam receber. Nesta perspectiva o cuidado de enfermagem fica centrado no doador, como usuário do sistema de saúde, transcendendo os conceitos de estado de saúde e de situação epidemiológica que apontam para o problema de saúde, que são referidos a indicadores objetivos e não definidos por sujeitos individuais ou coletivos ${ }^{(11)}$.

A Taxonomia das Necessidades de Saúde ${ }^{(8)}$ é composta por quatro grandes grupos que envolvem as 1) "Boas condições de vida", que poderiam ser entendidas tanto em um sentido funcionalista (fatores do "ambiente", fatores "externos") que determinam o processo saúde-doença, como nas formulações de autores marxistas que enfatizam os diferentes lugares ocupados por homens e mulheres no processo produtivo nas sociedades capitalistas, como as explicações mais importantes para os modos de adoecer e morrer, havendo consenso no que se refere a que a maneira como se vive, se "traduz" em diferentes necessidades de saúde; 2) As questões que circundam a discussão e o que se tornou um processo sobre o acesso e consumo da tecnologia de saúde capaz de melhorar e prolongar a vida, estão inscritas de forma não hierarquizada na tecnologia leve, leve-dura e dura; 3) A criação de vínculos afetivos e efetivos entre os usuários, neste caso, os doadores de sangue, e uma equipe ou profissional de saúde, em uma referência e relação de confiança, em que o profissional é o representante do sistema de saúde para o usuário, em uma relação contínua no tempo, pessoal e intransferível, com o encontro de subjetividades; 4) a necessidade de cada pessoa em ter graus crescentes de autonomia no seu modo de levar a vida. A reconceitualização dessa necessidade é que a informação e educação em saúde são apenas parte do processo de construção da autonomia de cada pessoa, e a autonomia implicaria a possibilidade de reconstrução, pelos sujeitos, dos sentidos de sua vida e esta ressignificação teria peso efetivo no seu modo de viver, incluindo aí a luta pela satisfação de suas necessidades, da forma mais ampla possível ${ }^{(8)}$.

A partir do contato da enfermeira com o doador de sangue na Triagem, onde é aplicado o questionário que tem como base o que é preconizado pelo Ministério da Saúde, e que busca informações clínicas e comportamentais, desde doações anteriores, problemas de saúde, uso e tratamento medicamentoso, traumático, e/ou cirúrgico; imunizações; histórico de doença pregressa e atual; hábitos de repouso; etilismo; tabagismo; uso de entorpecentes; hábitos 
sexuais; problemas neurológicos; contato com regiões epidêmicas e endêmicas; tatuagens e "pearcing"; condições de maternidade; aleitamento; aborto e período menstrual ou intercorrência ginecológica; e sobre o estilo de vida; além da vontade e voluntariedade para a doação em uma entrevista clínica epidemiológica e exame físico com avaliação hematimétrica, é possível o acesso a informações de sua vida e saúde, que tanto instrumentalizam a própria Triagem, como o cuidado do candidato.

O momento do encontro da enfermeira com o candidato a doador de sangue poderá ser mais que um mecanismo de exclusão e de minimizar os riscos para o serviço e para os receptores, poderá ser o início das relações interpessoais e de ajuda entre a enfermeira e os doadores de sangue idosos, e da criação dos vínculos (a) efetivos dos usuários com a equipe de saúde ou com o profissional.

Os idosos doadores de sangue são pessoas que podem conviver com doenças crônicas e degenerativas, e com estigmas sociais, culturais e econômicos relacionados ao envelhecimento, além da aceitação e adaptação pessoal e familiar a esta etapa da vida, e ainda se propõem, pelas mais diversas motivações, a doar um pouco do seu sangue, voluntária e altruisticamente, o que reforça sua cidadania e a auto-estima, que devem ser incentivados e preservados no cuidado da enfermagem em hemoterapia, na escuta das Necessidades de Saúde e nas interfaces das micropolíticas e da macropolítica do Sistema Único de Saúde.

Em uma descrição tradicional da fisiologia do envelhecimento, ou seja, do comprometimento natural dos órgãos e sistemas do corpo humano, os órgãos começam a diminuir a capacidade de exercerem suas funções, há declínio do processo de divisão celular, ocorrendo um balanço negativo, já que mais células morrem e menos são repostas. Aos poucos, o tecido muscular vai sendo substituído pelo adiposo. A pele fica mais fina e desidrata, com maior chance de lesar. Ocorre diminuição das acuidades auditiva e visual, comprometendo a função cognitiva desse idoso associada ao um possível afastamento do convívio social ${ }^{(3)}$.

Seguindo a linha desta compreensão do envelhecimento biológico, ocorre a diminuição da capacidade da medula óssea de responder às necessidades do corpo para células sanguíneas (hemácias, leucócitos, plaquetas). Essa incapacidade se deve a muitos fatores, incluindo a produção diminuída de fatores de crescimento necessários para a hematopoese pelas células estromais da medula. Assim, quando uma pessoa idosa necessita de mais células sanguíneas, a medula óssea pode não ser capaz de aumentar a produção dessas células adequadamente. A leucopenia ou anemia podem acontecer ${ }^{(12)}$. É inegável que mudanças sistêmicas e locais ocorrem no corpo como um todo.

Não poderemos nos furtar ao entendimento destas questões em suas interrelações políticas, sociais, econômicas e de saúde no cuidado à população. Este entendimento é um marco às questões gerenciais, de ensino e de planejamento para a prática nos próximos anos. É uma ação de e para o futuro na hemoterapia.

Profissionais capacitados e bem treinados a lidar com idosos no processo de doação de sangue serão os responsáveis por identificar as Necessidades de Saúde e por proceder ao cuidado que estas requeiram, tanto direta como indiretamente no momento do atendimento na Triagem Clínica em Hemoterapia, ou ao longo do processo de doação de sangue, com as respectivas orientações e resoluções a serem implementadas.

No cuidado direto de enfermagem aos doadores de sangue, ocorre a assistência direta ao cliente, relação entre subjetividades, relações interpessoais com o cliente em que a tecnologia leve se faz presente na constituição do trabalho vivo em ato; o cuidado indireto, voltado às atividades de supervisão e gerenciamento do cuidado direto, pode se estabelecer mais fortemente por via da tecnologia leve-dura, em muitas situações através do trabalho morto. O cuidado subjetivo, que envolve a tecnologia leve é relacionado às questões subjetivas, psicológicas, das relações interpessoais, do diálogo, do uso das palavras, das orientações e aconselhamentos, da presença que tranqüiliza, da confiança conforto - desconforto que o toque, o olhar, o odor e todos os sentidos desenvolvem quando cuidam, a leitura e interpretação da 
comunicação não-verbal e a ação que esta faz ser desenvolvida; diferentemente do cuidado objetivo que se relaciona às técnicas e condutas desenvolvidas com base em critérios e avaliações de sinais e sintomas palpáveis, numéricos, claros e objetivos típicos do trabalho morto e do emprego das tecnologias leve-dura e dura ${ }^{(9,13)}$.

Cuidar não é para ser confundido com significados, tais como: desejar o bem, gostar, confortar, e manter ou simplesmente ter interesse no que acontece com outra pessoa. Não é um sentimento temporário, tampouco uma questão de querer cuidar de alguma pessoa em especial, eventualmente. Cuidar é um processo, um modo de se relacionar com alguém que envolve desenvolvimento e cresce em confiança mútua, provocando uma profunda e qualitativa transformação no relacionamento $^{(14)}$.

O doador de sangue idoso, assim como qualquer outro doador, é o indivíduo que realiza a doação de uma unidade de sangue total ou um de seus componentes para ser administrado, por via endovenosa e com finalidade terapêutica, por profissional devidamente habilitado ao cliente que dele necessitar. O doador de sangue idoso, tanto está sujeito às inaptidões na triagem clínica, quanto as possíveis reações adversas ao processo de doação, algumas das reações adversas podem ser: a síncope vaso - vagal, lipotímia, convulsão, arritmia, taquicardia, choque hipovolêmico em instâncias graves, sudorese, palidez, ruptura da túnica média e externa do acesso venoso periférico com formação de hematoma, náuseas e vômitos, entre outras complicações ${ }^{(2,12)}$.

Como exemplo das inaptidões para a doação de sangue determinadas na Triagem apresentamos um levantamento realizado em um serviço de hemoterapia de um hospital universitário do Rio de Janeiro no período de janeiro de 2004 a maio de $2005^{(15)}$ que revela as dez primeiras causas de inaptidão tanto de adultos jovens, quanto de idosos (Valores percentuais em relação ao total de inaptidões 2.312- entre janeiro de 2004 e maio de 2005). A primeira delas é o Teor de Hemoglobina $(37,5 \%)$, seguida decrescentemente por Outras Causas (7\%); Uso de Medicamento (5,5\%);
Tatuagem, pearcing, maquiagem definitiva e acupuntura (3,9\%); Desistência (3,6\%); Hipertensão Arterial (3,5\%); Lesões de Pele $(2,9 \%)$; Manifestações Alérgicas Ativas (2,3\%); Estado Gripal (2,3\%); Hipotensão Arterial $(1,8 \%)$.

Dos dez itens listados anteriormente como causas de inaptidão de doadores de sangue, alguns podem representar riscos para os doadores, tanto durante o processo de doação, quanto posteriormente, e outros para os receptores, ou para ambos.

Cabe aos profissionais da Triagem orientar a população atendida sobre o processo de doação de sangue, seus riscos e benefícios, e procurar ouvir suas Necessidades de Saúde, visando à relação dialógica e de inter-relação no cuidado da clientela, e desmistificar a idéia de que por ser idoso estaria isento dos riscos de exposição a doenças sexualmente transmissíveis, ou por uso de drogas ilícitas, além, é claro, da própria condição fisiopatológica inerente ao processo de envelhecimento e dos tratamentos das comorbidades.

\section{ENVELHECER E DOAR SANGUE: CELEBRAÇÃO DA VIDA.}

É necessário auxiliar o idoso em seu processo de vida, na adaptação à realidade de manutenção e controle de suas patologias, fortalecendo suas relações pessoais, profissionais sociais, econômicas, culturais e afetivas, com (re-)integração à sociedade e família. Os profissionais das mais variadas especialidades, unidos em prol do idoso podem de fato favorecer esta conquista.

Por destinar-se a uma faixa etária a partir de 18 anos, a doação de sangue coloca as enfermeiras da Triagem, e o serviço de hemoterapia, como contribuintes para o envelhecimento saudável da população, uma vez que a cada encontro, deva ser realizada a entrevista para a doação de sangue, havendo a possibilidade de serem implementadas medidas educativas, de orientação, de encaminhamento e essencialmente prevenção de agravamentos de situações de saúde, contribuindo para o cuidado e o compromisso com a saúde da população ainda jovem, antes de atingirem os sessenta anos de idade. 
O cuidado de enfermagem, que tenha como perspectiva a saúde do doador, articulando a enfermagem hemoterápica, em um cuidado gerontológico, aos preceitos do SUS, preocupado com o doador idoso de sangue como ser político, social e humano favorece que os serviços de hemoterapia passem a contar com pessoas idosas. Nessa relação, os sabidamente doentes e os supostamente saudáveis, um doando sua vida para o outro, podem ter na enfermagem e nos profissionais dos serviços de hemoterapia intermediários desta conexão de vida. Nesse contexto, a enfermagem hemoterápica tem uma grande atuação e pode assumir o compromisso social e de saúde com a longevidade e cuidado da população.

Assim, é importante a reflexão sobre a essencialidade das campanhas para a doação de sangue, com o empenho para o esclarecimento, a sensibilização, educação e divulgação, particularmente para a população idosa, em que a doação de sangue é um ato de amor e respeito à vida. Trabalho que deve iniciar na formação do hábito do jovem adulto, enfocando a doação de sangue como um ato responsável, símbolo de autonomia, maturidade, e participação social a ser cultivado por toda a vida, em uma relação de cuidado e respeito dos cidadãos, e intermediada pela enfermagem hemoterápica.

\section{REFERÊNCI AS}

1. Instituto Brasileiro de Geografia e Estatística. Projeção da população do Brasil por sexo e idade para o período 1980-2050 - Revisão 2004. Brasília (Brasil): Ministério do Planejamento, Orçamento e Gestão; 2004.

2. Ministério da Saúde; Agência Nacional de Vigilância Sanitária. Resolução N N 153/2004 Determina o regulamento técnico para os procedimentos hemoterápicos. (Revoga as Resoluções RDC Nº 343 e 190). Brasília (Brasil): Ministério da Saúde; 2004.

3. Freitas EV, Py L, Cançado FAX, Gorzoni ML, Doll J. Tratado de geriatria e gerontologia. $2^{a}$ ed, Rio de Janeiro: Guanabara Koogan; 2006.

4. Beavouir S. A velhice. Rio de Janeiro: Nova Fronteira; 1990.

5. Ministério da Saúde. Lei № 8842/1994 Dispõe sobre a política nacional do idoso, cria o
Conselho Nacional do Idoso e dá outras providências. Brasília (Brasil): Ministério da Saúde; 1996.

6. Ministério da Saúde; Agência Nacional de Vigilância Sanitária. Resolução Nº 343 / 2002. Aprova o regulamento técnico para a obtenção, testagem, processamento e controle de qualidade de sangue e hemocomponentes para uso humano, que consta como Anexo I. Brasília (Brasil): Ministério da Saúde; 2002.

7. Benetti SRD, Lenardi MH. Significado atribuído ao sangue pelos doadores e receptores. Texto e Contexto Enfermagem. 2006; 15(1): 43-50.

8. Cecílio LCO. As necessidades de saúde como conceito estruturante na luta pela integralidade e eqüidade na atenção em saúde. In: Pinheiro $R$, Mattos $A$, organizadores. Os sentidos da integralidade na atenção e no cuidado à saúde. Rio de Janeiro: UERJ, IMS, ABRASCO; 2001. p. 113-126.

9. Merhy EE. Saúde: a cartografia do trabalho vivo em ato. 3a ed. São Paulo: Hucitec; 2007.

10. Conselho Federal de Enfermagem. Resolução $N^{\circ}$ 306/2006. Normatiza a atuação do enfermeiro em hemoterapia. Brasília (Brasil): Conselho Federal de Enfermagem; 2006.

11. Stotz EN. Necessidades de saúde: mediações de um conceito (contribuição das ciências sociais para a fundamentação teóricometodológica de conceitos operacionais da área de planejamento em saúde) [tese]. [Rio de Janeiro]: Escola Nacional de Saúde Pública/FIOCRUZ; 1991. 620p.

12. Smeltzer SC, Bare BG. Tratado de Enfermagem Médico-Cirúrgica. 9a edição. Rio de Janeiro: Guanabara Koogan; 2002.

13. Santos NLP. Serviço de hemoterapia privado: um espaço de cuidado dos clientes pelas enfermeiras [dissertação]. [Rio de Janeiro]: Escola de Enfermagem Alfredo Pinto/UNIRIO; 2002. 129 p.

14. Cianciarullo TI. Instrumentos básicos para o cuidar. São Paulo: Editora Atheneu; 2000.

15. Santos NLP, Nogueira CM, Silva SRA, Alves FP. Doação de sangue: a cidadania na terceira idade. Rev. Bras. Hematol. Hemoter. 2005;27(supl. 2): 263.

Artigo recebido em 14.08.07

Aprovado para publicação em 30.09.08 